\title{
Performance Analysis of Counter-Based Broadcast Scheme for Mobile Ad Hoc Networks
}

\author{
M. J. Lawali, M. Afshar Alam, and Harleen Kaur
}

\begin{abstract}
Broadcasting is a technique used for distributing of control packets in mobile ad hoc networks (MANETs), where source node diffuses a message to all other nodes in the network. Flooding is the simplest mechanism used for broadcasting in MANETs. Despite its simplicity, it can result in high redundant retransmission, contention and collision, a phenomenon called broadcast storm problem. In view of this, several schemes where proposed with aim to mitigate this problem, among which is the counter based scheme. It relies on counter threshold value for rebroadcast decision. The selection of an appropriate counter is crucial to the performance of the scheme. How, previous studies have focused on the choice of optimal counter threshold-value using network setting with very low traffic load. In this paper, we focus on the effect of the counter threshold value on the performance of counter-based scheme in terms of number saved rebroadcast nodes and reachability using Ns-2 simulation under varying condition of network density and traffic load. The results have shown that an optimal counter threshold is network density dependent.
\end{abstract}

Index Terms-Broadcasting, Flooding, Broadcast Storm Problem, Counter-based, Reachability.

\section{INTRODUCTION}

Mobile Ad hoc Networks (MANETs) are wireless networks formed by an autonomous system of mobile nodes that are connected via wireless links without using an existing network infrastructure or centralized administration. Such networks are suitable for scenarios which includes rescue/emergency operations in natural or environmental disaster areas, military operations, mobile conference, and home networking [1].

In MANETs, broadcasting plays a crucial role as a means of diffusing a message from source node to all other nodes in the network. It is a fundamental operation which is extensively used in route discovery, address resolution, and many other network services in a number of routing protocols [1]. For example, ad hoc on demand distance vector (AODV), dynamic source routing (DSR), zone routing protocol (ZRP), and location aided routing (LAR) use broadcasting or its derivative to establish routes. These protocols typically rely on simplistic form of broadcasting called flooding, in which each mobile node retransmits every unique received packet exactly once. Although flooding achieved high success rate in reaching all nodes in the network, it produces excessive redundant rebroadcast messages. In a dense network, this redundant rebroadcasts can often causes high contention and collision in the

Manuscript received February 4, 2001; revised October 9, 2011.

The authors are with the Department of Computer Science, Jamia Hamdard. (e-mail: muhammadlawali@yahoo.com, harleen_k1@rediffmail. com) network, leading to loss of precious bandwidth and battery power, a phenomenon called the broadcast storm problem $[2,3]$.

But in a high-density network, only a small number of broadcasts are needed to cover all the nodes in the whole network. This observation gives researchers the intuition and motive to design more effective broadcasting schemes. Specifically, new schemes are required to minimize the number of transmitting nodes for the sake of capacity and energy conservation, with the constraint that the number of covered nodes should not be reduced. Additionally, broadcasting schemes should be localized to satisfy the distributed feature of ad hoc routing protocols. Towards these requirements, a considerable number of schemes have been proposed in the literature [2-11]. These schemes are commonly divided into two categories: deterministic scheme[2,6] and probabilistic schemes [5,8]. Deterministic schemes use network topological information to build a virtual backbone that covers all the nodes in the network. To build a virtual backbone, nodes exchange information about their immediate or two hop neighbours. However, this often incurs large overhead in terms of time and message complexity for building and maintaining the backbone, especially in the presence of mobility. Probabilistic schemes, in disparity, rebuild a backbone from scratch during each broadcast[5]. Nodes make instantaneous local decisions about whether to broadcast a message or not using information derived only from overheard broadcast messages. Consequently these schemes incur a smaller overhead and demonstrate superior adaptability in changing environments when compared to deterministic schemes [8]. However, these schemes have poor reachability as a tradeoff against overhead.

An efficient broadcast should be able to minimize the number of retransmissions without sacrificing reachability or having any significant degradation. In this paper, we examine counter-based broadcast scheme as one of the proposed probabilistic schemes in literature that mitigate the broadcast storm problem inherent with flooding. Counterbased broadcast schemes for MANETs have been first proposed in [2] and further investigated in [3,4,5]. In counter-based schemes, every mobile node relies on a predetermined counter threshold-value $(C)$, to decide whether or not to rebroadcast a packet. These schemes do not require global topological information of the network in order to make rebroadcast decision. Thus, these schemes are localized and can considerably reduce the number of retransmission predominant in flooding but on the other hand cannot guarantee full network coverage in sparse network. One major challenge in counter-based schemes is how to select an appropriate $C$ that can optimize the performance of counter-based scheme in terms of number of 
retransmitting nodes, reachability and end-to-end delay. Most counter-based schemes assumed a counter thresholdvalue of 3 or 4 [5,8]. It has been shown in [2] that a threshold-value of 3 or 4 can save many rebroadcasts in a dense network while achieving a reachability ratio comparable to flooding. On the other hand, a larger threshold of $C>6$ will provide less saving of rebroadcasts in a sparse network but behave almost like flooding in terms of reachability. However, the studies in [2,4] have determined the threshold-value in the context of light traffic injection rate scenario.

Several previous studies $[4,5,6]$ have used the threshold value suggested in[2]. Nevertheless, these studies have assumed different traffic conditions (i.e. 10 packets per second and above) that have not been considered in [2]. This paper investigate the effect of different counter threshold values on the performance of counter-based broadcast schemes in MANETs under a wide range of traffic conditions (i.e. low, moderate and high traffic loads) and network density. Simulation results reveal the sensitivity of the performance of the counter-based broadcast scheme on the selection of the counter threshold-value under different traffic conditions. Moreover, we will show that when factors such as traffic and density are taken into account, the optimal counter threshold value can be different from that reported in [2].

The rest of the paper is organized as follows. Section 2 introduces a review of the counter-based broadcast scheme as well as some related works. Performance analysis is presented in Section 3 and finally, section 4 is our concluding remarks and some suggestion of future directions.

\section{REVIEW OF THE COUNTER-BASED SCHEME}

Counter-based scheme was introduced in [2] after analysing the additional coverage of each rebroadcast when receiving $n$ copies of the same packet. The predefined threshold $\mathrm{C}$ is the key parameter in this approach. They showed that about $67 \%$ of the rebroadcasts could be saved when choosing a $\mathrm{C}$ value of 3 or 4 while the amount of saving decreases sharply if $C>6$, especially in sparse network. In their follow-on work [4], the authors have proposed an adaptive counter-based scheme in which each node can dynamically adjust its threshold value $C$ based on its number of neighbours. Specifically, they extend the fixed threshold $C$ to a function $C(n)$, where $n$ is number of neighbours of the node. This approach requires a neighbour discovery mechanism to estimate the current value of $n$ which can be achieved through periodic exchange of 'Hello' packets among mobile nodes.

The counter-based scheme can be expressed as follow: a node initiates a counter with a value of one and sets a RAD (random chosen between 0 and $T_{\max }$ seconds) upon reception of previously unseen packet. During the RAD, the counter is incremented by one every time it receives a redundant packet. The delay is necessary for two reasons. First, it allows nodes adequate time to receive redundant packets and assess whether to rebroadcast. Second, the randomized scheduling prevents collision [6].If the counter is less than the threshold $(\varepsilon \in \mathbb{E}, \varepsilon \geq 2)$ upon the expiration of the RAD timer, the node simply retransmits the packet. Otherwise it shall drop it and decline to retransmit it.

Other variants of counter-based scheme include a dynamic probabilistic broadcast [5] scheme and the distance-aware counter-based scheme [7]. The main idea of the first scheme is the combination of the probabilistic and counter-based approaches. The scheme is implemented for route discovery process using AODV as base routing protocol. The rebroadcast probability $P$ is dynamically adjusted according to the value of the local packet counter at each mobile node. Therefore, the value of $P$ changes when the node moves to a different neighbourhood. The packet counter is used as density estimates (i.e. a high value implies that the number of neighbours is high, and a low value corresponds to a small number of neighbours). This scheme uses the counter threshold value suggested in [2], however, a different traffic rate of 10 packet per second was used for the evaluation of the scheme. The distance-aware counterbased broadcast scheme called "DIS_RAD introduces the concept of distance into counter-based broadcast scheme. The scheme gives nodes closer to the border of the transmission range a higher rebroadcast probability because they can have a high chance of reaching more nodes. A distance threshold is employed to distinguish between interior and border nodes using two distinct RAD values with the border nodes having shorter RADs than the interior nodes. This simple adaptation provides border nodes with higher rebroadcast probability and a lower rebroadcast probability for the interior nodes. Although the approach has superior performance over traditional counter-based scheme it suffers from the limitation of all distance-based schemes (i.e. determination of location information and optimal threshold value). Moreover, the same counter threshold was used as in the previous scheme which might not be optimal in the considered settings.

In a related work, [8] has proposed a colour-based broadcast scheme in which every broadcast message has a colour-field, with a rebroadcast condition to be satisfied after expiration of the timer similar to counter-based scheme. A node rebroadcasts a message with a new colour assigned to its colour-field if the number of colours of broadcast messages overheard is less than a colour threshold $\mu$.

Recently, an enhanced counter-based scheme (ECS) [10] was proposed which combines the merits of probabilitybased and counter-based algorithms. The scheme enables mobile nodes to make localized rebroadcast decisions on whether or not to rebroadcast a message based on counter threshold and forwarding probability values of around 0.5 to yield a better performance in terms of saved-rebroadcast, end-to-end delay and reachability. However, its use of single fixed forwarding probability for all nodes in the network regardless of whether the node is in sparse or dense region of the network has make it inflexible in a typical MANET scenario where regions of varying node density co-exist in the same network. In their follow-on work a new adjusted counter-based broadcast scheme (ACBS) [11] is proposed, which uses two rebroadcast probabilities to differentiate between mobile nodes in sparse and dense area.

However, most of these proposed counter-based broadcast schemes used the threshold value suggested in [2] 
for the evaluation of their scheme which might not be optimal in their network settings.

\section{Performance Analysis}

This section describes the details of our simulation environment, performance metrics used in our analysis and finally simulation results.

\section{A. Simulation setup}

The study is conducted using Ns-2 simulator - a discrete event simulator [12] widely used in MANETs community [6-11,13,14], to simulate a network of $1000 \times 1000 \mathrm{~m}^{2}$ area populated with $20,40,60, \ldots, 200$ mobile nodes. The simulation model consists of two set of scenario files: topology scenario and traffic generation files. The topology scenario files define the mobility model which governs the distribution of mobile nodes within the simulation area over the simulation period. On the other hand, the traffic pattern file contains information such as packet type, data packet size, broadcast packet origination rate and the number of traffic flows. In all scenarios, each node is assumed to be equipped with a wireless transceiver operating on IEEE 802.11b wireless standard [15]. The physical radio characteristics of each node such as the transmitting power, signal to noise and interference ratio and antenna gain, are chosen to mimic the commercial Lucent's OriNOCO Wireless LAN PC Card [16] with a nominal bit rate of $11 \mathrm{Mb} / \mathrm{s}$ and a transmission range of 100 meters with an Omni-directional antenna. To gain more realistic signal propagation than with the deterministic free space or tworay ground reflection models [17], the shadowing model is used as a radio propagation[18]. The simulation is allowed to run for 900 seconds for each simulation scenario to avoid immature termination and to keep the simulation time manageable. The random trip mobility model [19] was used as the mobility model to generate 30 mobility topologies. Although, random way-point model [20] is the most widely used mobility model but it suffers from speed decay problem, as such it takes more time to reach a stable distribution of mobile nodes. In random trip model, at a trip transition instant, a mobile node picks a trip destination uniformly at random within the area and samples numeric speed from a uniform distribution [minimum speed, maximum speed]. At the end of the trip, the mobile node picks another path according to the model's trip selection rule driven by a Markov chain. This cycle repeats until the end of the simulation time. Unlike other random mobility model, random trip node mobility distribution converges to a steady-state regime from origin of an arbitrary trip and there is no need to discard initial sets of simulation observations. Other simulation parameters that have been used in our experiment are shown in Table I.

This paper focus on two major network operating conditions: network density and traffic load, using two different cases by varying one condition while keeping the other constant in order to avoid the effect of one condition on the performance result of the varying condition. The mobility is assumed to be constant in order to avoid the effect of mobility in terms of frequent link breaks and also to focus on one category of node speed that can mimic a slow walking human (i.e. $2 \mathrm{~m} / \mathrm{s}$ ).
TABLE I: SUMMARY OF SIMULATION PARAMETERS

\begin{tabular}{|l|l|}
\hline Simulation Parameter & Value \\
\hline Simulator & NS-2 $(2.30)$ \\
Transmission range & $100 \mathrm{~m}$ \\
Packet size & 512 bytes \\
Interface queue length & 50 \\
Topology size & $1000 \mathrm{~m} \times 1000 \mathrm{~m}$ \\
Number of nodes & $20,40, \ldots, 200$ \\
Simulation time & 900 seconds \\
Bandwidth & $11 \mathrm{Mbps}$ \\
Maximum speed & $1,5,10, \ldots 20 \mathrm{~m} / \mathrm{s}$ \\
Packet origination rate & $1,10,20, \ldots 50$ packet/sec \\
Number of trials & 30 \\
Confidence interval & $95 \%$ \\
MAC type & $802.11 \mathrm{~b}$ \\
Counter threshold & $2-6$ \\
Traffic type & Constant bit rate (CBR) \\
\hline
\end{tabular}

- Network Density: This refers to the total number of nodes in the network. It is used to study the effect of varying network density on the performance of the network. The simulation area is kept constant in all scenarios from sparse to dense network. Simulation has been performed by deploying 20, 40, 60, ..., 200 nodes while fixing the maximum speed to $2 \mathrm{~m} / \mathrm{s}$ and the traffic load of 10 packet per second

- Traffic Load: This is used to study the effect of varying the amount of traffic load on the performance of the network. Broadcast injection rate of 10, 20 packets per second were used while the network density is kept to 100 nodes to avoid sparse and dense scenarios with a maximum speed $2 \mathrm{~m} / \mathrm{s}$ to avoid the effect of mobility.

\section{B. Performance measures}

The performance of different threshold values is measured using the following performance metrics which have been widely used in the literature [2-11, 13, 14].

- Retransmitting nodes: The number of nodes in the network that receives a broadcast packet and rebroadcast it.

- Reachability (RE): The percentage of network mobile nodes that receive a given broadcast packet over the total number of nodes that is reachable, directly or indirectly.

\section{Simulation and Discussion}

This section presents the performance results of the two network operating conditions (i.e., density and load) on counter-based scheme over different threshold values. The simulation output is collected using replication mean method[21] where each data point represents an average of 30 different randomly generated mobility topologies using 95\% confidence intervals.

\section{1) Effect of Network Density}

The network density has been varied by deploying 20, 40, $60 \ldots 200$ nodes over a network topology of $1000 \mathrm{~m}$ x 1000m. Each node in the network moves according to random trip mobility model with minimum and maximum speeds of $1 \mathrm{~m} / \mathrm{s}$ and $2 \mathrm{~m} / \mathrm{s}$ respectively. In each simulation trial, a broadcast injection rate of 10 packets per second has been used with each new broadcast packet assigned a source node 
randomly chosen from the entire pool of network nodes in order to create a random traffic pattern.

\section{Number of Retransmitting Nodes}

Figure 1 shows the effects of density on the performance of different counter threshold values together with flooding in terms of number of retransmitting nodes. The figure reveals that the number of retransmitting nodes for a given threshold value increases with increasing network density. A low threshold value (i.e. $C=2$ ) requires least number of retransmissions while those utilising higher threshold values (i.e. $C=5,6$ ) require the largest number of rebroadcasts. In fact threshold values greater than 4 behave almost similar to flooding because most of the nodes retransmit the packets. For example in Figure 1, for a network of 100 nodes about $40 \%$ of the nodes retransmit for the threshold value 2 while around $98 \%$ of the nodes retransmit for threshold value 6 .

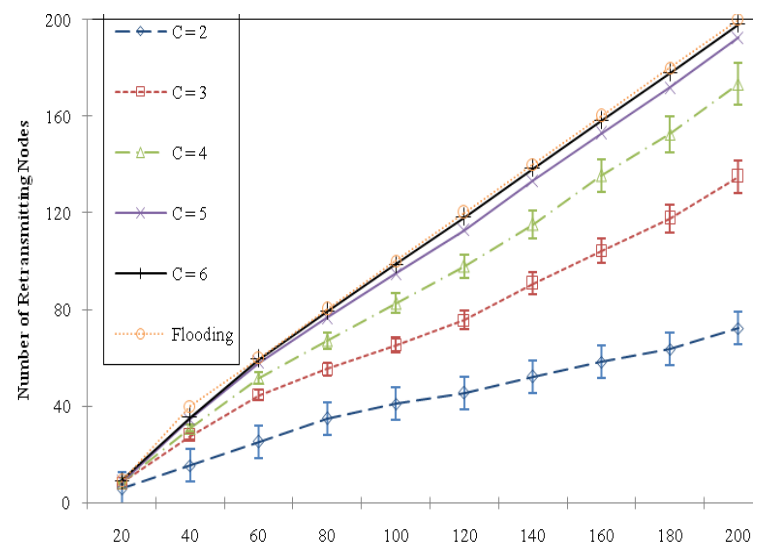

Fig. 1. Number of retransmitting nodes vs. number of nodes placed over $1000 \mathrm{~m} \times 1000 \mathrm{~m}$ area when a broadcast rate of 10 packets/sec is used for different threshold values.

\section{Reachability}

Figure 2 depicts the reachability performance achieved by the different threshold values over a varying network density. The figure shows that reachability increases with increase in network density. For example, reachability achieved by threshold value 2 increases from $26 \%$ for 20 nodes to $98 \%$ for 100 nodes while that of threshold value 6 increases from $45 \%$ to $99.9 \%$ for 20 and 100 nodes respectively. This is because as number of nodes increases there is more likelihood that nodes are located within the transmission range of each other and thus resulting in a better network connectivity. Similarly, the figure also reveals that low threshold value (i.e. $C=2$ ) achieves the least reachability in sparse to medium networks (20 to 80 nodes). But as the density increases reachability improves for all threshold values. As in Figure 2, for threshold values 4 and above, the counter-based scheme converges to flooding in terms of reachability performance. This is because the higher the threshold values, more nodes retransmit the broadcast packets. Therefore, to maintain a high reachability in sparse networks, a higher threshold value is required while to maintain reachability in dense networks, a low threshold value can be used. Thus, reachability improves with increased network density.

\section{2) Effect of Traffic Load}

To investigate the impact of traffic load, the injection rates of $1,10,20,30,40$ and 50 packets per second have been used with each new broadcast packet assigned a source node randomly chosen from the entire pool of network nodes. 100 nodes are placed over a network topology of $1000 \mathrm{~m} \times 1000 \mathrm{~m}$ area and each node in the network moves according to random trip mobility model with minimum and maximum speeds of $1 \mathrm{~m} / \mathrm{s}$ and $2 \mathrm{~m} / \mathrm{s}$ respectively.

\section{Number of Retransmitting Nodes}

The results in Figure 3 show the effects of offered traffic load on the network performance for different threshold values in terms of number of retransmitting nodes. As expected, the number of retransmitting nodes for a given threshold value almost remain constant over different traffic loads. This is due to the use of fixed number of nodes (i.e. 100 nodes) in this simulation scenario. Nevertheless, a low threshold value (i.e. $C=2$ ) requires the least number of retransmissions while high threshold values (i.e. $C=5,6$ ) require the largest number of retransmissions. For example in the same figure, around $41 \%$ (41 nodes) of nodes retransmit when $C=2$ while about $65 \%$ of the nodes retransmit for $C=3$ and around $84 \%, 94 \%$ and $98 \%$ of the nodes retransmit for $C=4,5$ and 6 respectively. Therefore, the higher the threshold values the higher the number of retransmitting nodes.

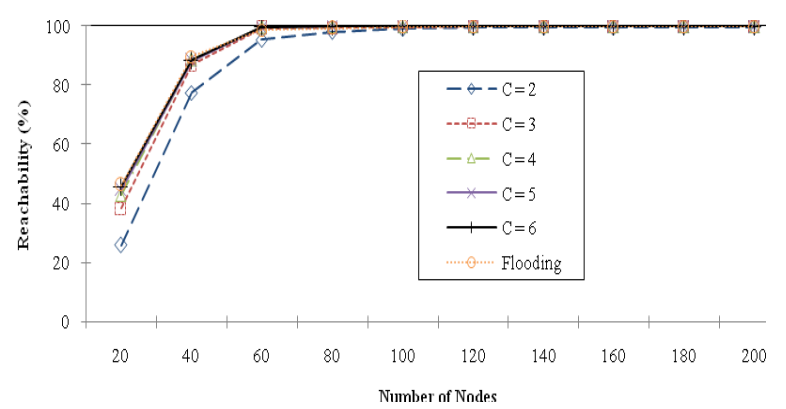

Fig. 2. Reachability vs. number of nodes placed over $1000 \mathrm{mx} 1000 \mathrm{~m}$ area when a broadcast rate of 10packets/sec is used for different threshold values.

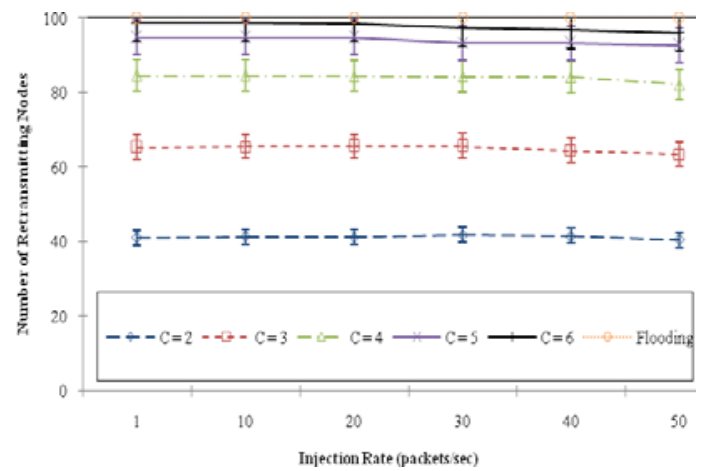

Fig. 3. Number of retransmitting nodes vs. broadcast injection rates in a network of 100 nodes placed over $1000 \mathrm{mx} 1000 \mathrm{~m}$ area for different threshold values.

\section{Reachability}

Figure 4 reveals that reachability decreases with increased broadcast injection rate, i.e. a heavier load will result in a lower reachability performance. This is true for all threshold values and flooding, because a high broadcast rate leads to more contention and collision among broadcast packets. For example, flooding is the most affected as reachability falls to around $85 \%$ at a broadcast rate of 50packets/sec. Moreover, to maintain a better reachability a low threshold value is required especially in dense network. The figure also reveals that a low threshold value is advantageous when the injection rate is over 20 packets per second. 


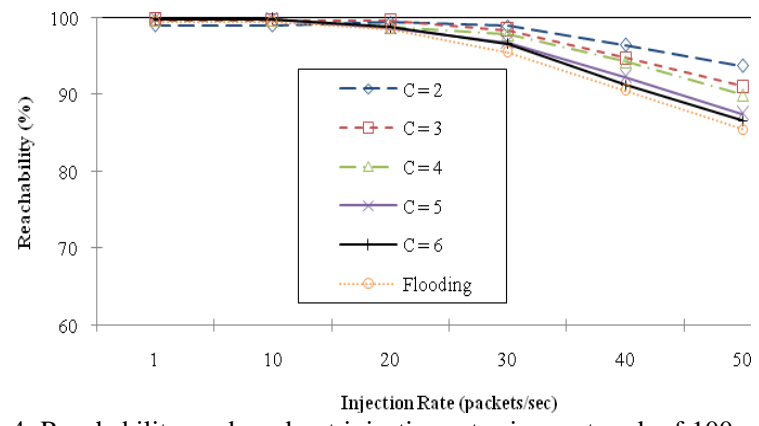

Fig. 4. Reachability vs. broadcast injection rates in a network of 100 nodes placed over $1000 \mathrm{mx} 1000 \mathrm{~m}$ area for different threshold values.

\section{CONCLUSIONS AND FUTURE WORK}

This paper has conducted a performance analysis of counter-based broadcast scheme to assess the effects of network density and traffic load over different counter threshold values. The results have revealed that network density and offered traffic load have significant impact on the performance of the scheme in terms of number of retransmitting nodes and reachability. Furthermore, the results have shown that the selection of an appropriate threshold value dictates the achieved performance output of counter-based scheme. However, it has been observed that the performance of counter-based is optimised when counter threshold values of 2 or 3 is used in increasing network density and traffic load.

The performance of counter-based scheme can be enhanced by adapting the counter threshold values to local density. So that sparse and dense regions of the network can use different threshold values to improve performance. However, this adaptation requires the exchange of neighbourhood information between nodes using periodic exchange of "hello" packets. Similarly, the reachability performance of counter-based scheme suffers in congestive network irrespective of which threshold value is used. This is particularly due to the scheme's random assessment delay (RAD) mechanism. However, a possible solution to this problem is to devise a mechanism which can adapt node's RAD to its local congestion level.

\section{REFERENCES}

[1] M. D. Colagrosso, "Intelligent broadcasting in mobile ad hoc networks: Three classes of adaptive protocols," EURASIP Journal on Wireless Communication and Networking, vol. 2007, pp. Article ID 10216, 16 pages, 2007.

[2] S. Ni, Y. Tseng, Y. Chen, and J. Sheu., "The broadcast storm problem in a mobile ad Hoc networks," in Proceeding of the ACM/IEEE International Conference on Mobile Computing and Networking (MOBICOM), 1999, pp. 151-162.
[3] Y.C. Tseng, S.Y. Ni, Y.S. Chen and J.P. Sheu, "The broadcast storm problem in a mobile ad hoc network," Wireless Networks, 8, 153-167, 2002.

[4] Y.C. Tseng, S.Y. Ni And E.Y. Shih, "Adaptive approaches to relieving broadcast storms in a wireless multihop ad hoc networks," IEEE Transaction on Computers, 52, 545-557, 2003.

[5] Q. Zhang and D. P. Agrawal, "Dynamic Probabilistic Broadcasting in MANETs," Journal of Parallel and Distributed Computing, vol. 65, pp. 220-233, 2005.

[6] B. Williams and T. Camp, "Comparison of Broadcasting Techniques for Mobile Ad Hoc Networks," in Proceeding MOBIHOC. Lausanne, Switzerland: ACM, 2002, pp. 194-205.

[7] C. Chen, C.K. Hsu and H.K. Wang,” A distance-aware counter-based broadcast scheme for wireless ad hoc networks," Military Communications Conference (MILCOM 2005) IEEE, 2005.

[8] A. Keshavarz-Haddad, V. Ribeiro, and R. Riedi, "Color-Based Broadcasting for Ad Hoc Networks," in Proceeding of the 4th International Symposium on Modeling and Optimization in Mobile, Ad Hoc, and Wireless Network (WIOPT' 06). Boston, MA, 2006, pp. 1 - 10.

[9] J. Cartigny and D. Simplot, "Border node retransmission based probabilistic broadcast protocols in ad hoc networks," Telecommunication Systems,, vol. 22, pp. 189-204, 2003.

[10] A. Mohammed, M. Ould-Khaoua, and L. Mackenzie, "An Efficient Counter-Based Broadcast Scheme for Mobile Ad Hoc Networks," in Proceedings of the Fourth European Performance Engineering Workshop (EPEW 2007) Lecture Notes in Computing Science Volume 4748, K. Wolter, Ed. Berlin, Germany: Springer-Verlag 2007, pp. $275-283$.

[11] A. Mohammed, M. Ould-Khaoua, L. Mackenzie and J. Abdulai, “ An Adjusted Counter-Based Broadcast Scheme for Mobile Ad Hoc Networks," Proceedings of the Tenth International Conference on Computer Modeling and Simulation (EUROSIM/UKSIM 2008).

[12] The Network Simulator ns-2, Available from: http://www.isi.edu/nsnam/ns/

[13 ] J. Broch, D.A. Maltz, D.B. Johnson, Y.C. Hu and J. Jetcheva, “A Performance Comparison of Multi-Hop Wireless Ad Hoc Network Routing Protocols," Proceedings of ACM/IEEE International Conference on Mobile Computing and Networking (MOBICOM'98), $85-97,1998$.

[14] P. Rogers, and N. Abu-Ghazaleh, "Towards reliable network wide broadcast in mobile ad hoc networks,"[online],Available from: http://arxiv.org/abs/cs/0412020.

[15] IEEE std 802.11b, Wireless LAN Medium Access Control (MAC) and Physical Layer (PHY) Specifications. Higher-Speed Physical Layer Extension in $2.4 \mathrm{GHz}$ Band. Supplement to IEEE 802.11 Standard, 1999.

[16] Lucent Technology-Bell Labs Innovation, ORiNOCO PC Card(2000) User's Guide. [online] Available from: ftp://ftp.orinacowireless.com /pub/docs/ORINOCO/MANUALS/ug_pc.pdf.

[17] T.K. Sakar, Z. Ji, K. Kim, A. Medourand and M. Salazar-Palma, "A survey of various propagation models for mobile communications," Proceedings of IEEE Antennas and Propagation Magazine, 45, 51-82, 2003.

[18] T.S. Rappaport, "Wireless Communications: Principles and Practice”New Jersey, Prentice Hall PTR Upper Saddle River, 2001.

[19] J.Y, L. Boudec and M. Vojnovic, "The random trip model: stability, stationary regime, and perfect simulation," IEEE/ACM Transactions on Networking, 14, 1153-1166, 2006.

[20] J. Boleng,"Normalizing mobility characteristics and enabling adaptive protocol for ad hoc networks," Proceedings of the IEEE Local and Metropolitan Area Networks Workshop (LANMAN), 2001.

[21] A. M. LAW," Simulation Modeling and Analysis," New Delhi, Tata McGraw-Hill Publishing Company Limited, 2008. 